\title{
The Mental Health of Healthcare Staff Working During the COVID-19 Crisis: Their Working Hours as a Boundary Condition
}

\author{
Haitong Gong' \\ Stephen X Zhang (iD ${ }^{2}$ \\ Khaled Nawaser (iD ${ }^{3}$ \\ Asghar Afshar Jahanshahi (iD) ${ }^{4,5}$ \\ Xingzi Xu iD ${ }^{6}$ \\ Jizhen Li (iD) ${ }^{6}$ \\ Afsaneh Bagheri (iD ${ }^{7}$ \\ 'Collegue of Nursing and Health \\ Sciences, Flinders University, Adelaide, \\ SA, Australia; ${ }^{2}$ Faculty of Professions, \\ University of Adelaide, Adelaide, SA, \\ Australia; ${ }^{3}$ Universidad Católica Los \\ Ángeles de Chimbote, Instituto de \\ Investigación, Chimbote, Peru; \\ ${ }^{4}$ CENTRUM Católica Graduate Business \\ School (CCGBS), Lima, Peru; ${ }^{5}$ Pontificia \\ Universidad Católica del Perú (PUCP), \\ Lima, Peru; ${ }^{6}$ School of Economics and \\ Management, Tsinghua University, Beijing, \\ People's Republic of China; ${ }^{7}$ Faculty of \\ Entrepreneurship, University of Tehran, \\ Tehran, Iran
}

Purpose: Healthcare staff operate at the forefront of the fight against COVID-19 and hence face enormous physical and mental pressures. We aim to investigate healthcare staff's mental health issues and the associated predictors during the COVID-19 pandemic. Specifically, this paper aims to identify some unique predictors of healthcare staff's mental health issues in Iran, the second country after China to experience a major COVID-19 crisis.

Methods: An online survey of 280 healthcare staff in all 31 provinces of Iran assessed staff's mental distress (K6), depression, and anxiety (PHQ-4) during April 5-20, 2020 during the COVID-19 crisis.

Results: Nearly a third of healthcare staff surpassed the cutoff for distress, depression, and anxiety symptoms. Females or more educated healthcare staff were more likely to experience distress. Those who were unsure whether they had COVID-19 were more likely to experience distress and depression symptoms. The number of COVID-19 cases among a healthcare worker's colleagues or friends positively predicted the worker's anxiety symptoms. Amongst healthcare staff, doctors were less likely than radiology technologists to experience distress and anxiety symptoms. Technicians and obstetrics staff experienced fewer anxiety symptoms. The age and the weekly working days of healthcare staff interacted such that age is asignificant predictor of mental health issues among younger but not older healthcare staff. Conclusion: The identification of the predictors of mental health issues can guide healthcare organizations to screen healthcare workers who are more likely to be mentally vulnerable in the ongoing COVID-19 pandemic.

Keywords: healthcare staff, mental health, working time, age, COVID-19, epidemic, Iran

\section{Introduction}

Healthcare staff have been under enormous physical and mental pressures during the COVID-19 pandemic. $^{1-6}$ Working under the COVID-19 pandemic exposes many healthcare staff to the risk of being infected with the virus, difficult work environment, tremendous workloads, shortage of equipment such as PPE, and "moral injury", which refers to the distressing awareness that healthcare workers face when they cannot meet all the needs of their patients. ${ }^{7}$ Many healthcare workers also experience stress from either the risk of infecting their family members by bringing the virus home or from the isolation by staying away from their families. ${ }^{8,9}$ All of these work and health-related issues can harm workers' mental health. $^{10,11}$ Indeed, some healthcare workers have suffered mentally and even committed suicide due to the enormous stress of the COVID-19 pandemic. $^{12}$
Correspondence: Stephen X Zhang Faculty of Professions, University of Adelaide, 9-28 Nexus 10 Tower, 10

Pulteney St, Adelaide, SA, 5000, Australia Tel +61 8831 39310

Email stephen.x.zhang@gmail.com 
Such issues have been documented in several countries. ${ }^{1,13}$ This paper aims to investigate healthcare staff's mental health issues and their associated predictors in Iran, which was the second country after China to experience a major COVID-19 outbreak in early 2020 . $^{3,5,14,15}$

We identify the predictors of mental issues using relatively assessable variables, especially the sociodemographic ${ }^{16,17}$ clinical, and work-related characteristics of healthcare staff. ${ }^{18,19}$ Specifically, our study represents one of the first attempts to use the clinical and the working characteristics $^{20}$ of healthcare staff, ${ }^{6,21}$ such as their job functions and their number of working days per week, to identify mental issues. We also examine the interaction between healthcare workers' weekly working days and age above and beyond the prior literature on the risk factors of mental disorders among healthcare staff. ${ }^{13-15}$ Our approach of identifying risk factors based on workrelated characteristics can help healthcare organizations to better identify healthcare staff who are more likely to suffer mentally and may need mental health services during the ongoing COVID-19 pandemic and contribute new predictors to the burgeoning research on mental health of healthcare workers under COVID-19.,

\section{Methods}

Our study surveyed healthcare staff in Iran during April 5-20, 2020 when Iran was experiencing a crisis of COVID-19. On April 5, the date the survey started, a total of 58,226 cumulative COVID-19 cases had been diagnosed in Iran and 2483 new cases were diagnosed. Of the diagnosed cases, 3,603 total deaths were reported in a day, 43 of whom were healthcare staff. ${ }^{17}$ On April 20, the date the survey ended, a total of 83,505 cumulative COVID-19 cases had been reported in Iran with 1,294 daily new cases and 5,209 total deaths. These counts were among the highest across all countries in April 2020, and the COVID-19 crisis in Iran caused extreme strain on medical resources and great psychological pressures on Iranian healthcare staff. ${ }^{13}$

\section{Study Design}

This study followed the American Association for Public Opinion Research (AAPOR) reporting guideline. All the participants consented to the survey before their enrolment. The survey followed Declaration of Helsinki and was approved by the ethics committee at Tsinghua University (\#20200304). The cover letter of the survey briefed the participants about the objectives of the study, the procedure, and the anonymous and voluntary nature of the survey. All the participants completed the survey voluntarily and anonymously, and were free to leave the survey at any time. To access more participants under the severe constraints of the COVID-19 pandemic, we shared the links to the survey on social media channels (Instagram, WhatsApp, and Telegram) ${ }^{22}$ and requested that healthcare staff share the survey with their colleagues. The final sample comprised 280 healthcare staff across all 31 provinces of Iran.

\section{Measures}

\section{Predictors}

Our predictors include several relevant socio-demographic, clinical, and work-related characteristics. Socio-demographic characteristics include healthcare workers' age (in years), gender (male or female), education level (categorical variable: $1=$ below a diploma or 12 years of education, 2 = a diploma with 12 years of education, $3=$ a student or a graduate of a 2year college degree, $4=$ a student or a graduate of a 4-year college degree, $5=$ a student or a graduate of a master degree, and $6=$ a student or a graduate of a doctoral degree).

The clinical variables include the healthcare workers' chronic health issues (no, unsure, yes), COVID-19 infection status (no, unsure, yes), and the number of colleagues or friendspositive with COVID-19. Drawing on previous research finding that people who have comorbidity may experience more mental health disorders in the COVID-19 pandemic, ${ }^{23}$ we asked the participants in this survey whether they had any chronic health issues. The participants were also asked whether they had been infected with COVID-19 within the past two weeks. Lastly, the participants reported how many of their colleagues or friends had tested positive with COVID-19 within the past two weeks.

The work-related variables consist of healthcare staff's job functions, whether their work institution is private or public, and the number of working days a week. Because healthcare staff are exposed to COVID-19 to a different extent depending on their job functions, ${ }^{19}$ we asked the healthcare staff to indicate their job functions (categorical variable: $1=$ doctors, $2=$ nurses, $3=$ technicians, $4=$ radiology technologists, $5=$ medical students/interns, $6=$ healthcare administrators, $7=$ supporting/facility/cleaning staff, $8=$ volunteers, $9=$ obstetricians). We also asked the participants whether their healthcare institutes are private or public and how many days they had worked in the prior week. All the measures were developed in English and then we either found existing Farsi version of the 
instruments when available or translated them into Farsi, the official language of Iran.

\section{Outcome Variables}

The three mental health variables comprise distress, depression, and anxiety. We assessed distress by the Kessler Psychological Distress Scale (K6) with Cronbach's alpha of $0.79 .{ }^{20}$ We measured depression and anxiety by the 4-item Patient Health Questionnaire (PHQ4 , with a score of $0-12$ ), which is an ultra-brief scale of a 2-item depression scale (PHQ-2) and a 2-item anxiety scale (GAD-2). Given these scales had been translated and validated in previous studies conducted in Iran, we used the Farsi versions of the measures. ${ }^{24}$ The cutoff scores for detecting symptoms of distress, depression, and anxiety were 13,3, and 3, respectively. Participants who scored higher than the cutoff thresholds were characterized as having symptoms of mental health issues.

\section{Statistical Analysis}

Our empirical analysis was performed using Stata 16.1 software. The statistical significance level was assessed by $p<0.05,2$-tailed. Multivariable logistic regression was used to determine the potential risk factors of distress, depression, and anxiety. The statistical regression results showed the associations between the risk predictors and the outcome variables with odds ratios (ORs) and their 95\% confidence intervals (CIs). We also presented a margin analysis to further investigate the interaction effect between the healthcare workers' age and number of working days.

\section{Results}

\section{Descriptive Statistics}

Table 1 displays the descriptive characteristics of the 280 healthcare staff. Among them, 21.4\% (60), 30.0\% (84), and $32.9 \%$ (92) surpassed the cutoff for distress, depression, and anxiety. The majority of the healthcare workers $(60.0 \%, 168)$ were female. Most of the participants $(70.5 \%, 196)$ were under 40 years old, and $79.4 \%$ (239) of the participants had university degrees or 2-year diplomas. Most of the healthcare workers $(80.3 \%, 226)$ did not have chronic health issues, $2.5 \%$ (7) reported they were COVID-19 positive, while $27.1 \%$ (76) reported they were unsure whether they had COVID-19. A little more than half of the healthcare workers $(53.0 \%, 161)$ reported they had colleagues or friends who were COVID-19 positive with a mean of 3.9 (min: 0; max: 150). Given the number of COVID-19 positive colleagues or friends is a count variable, we transformed it by taking its logarithm.

In terms of job function of the healthcare staff, $15.0 \%$ (42) were doctors, $20.4 \%$ (57) were nurses, $9.0 \%$ (25) were technicians, $22.1 \%$ (62) were radiology technologists, 3.6\% (10) were medical students and interns, $13.2 \%$ (37) were healthcare administrators or interns, $3.6 \%$ (10) were supporting staff (ie, facility or cleaning staff), $10.3 \%$ (29) were obstetrics staff, and 2.8\% (8) were volunteers. About three-quarters of the healthcare staff worked in a public healthcare organization $(74.7 \%, 223)$, and the majority of them worked at least three days a week $(72.2 \%, 202)$.

\section{Logistic Regression Results}

Table 1 presents the multivariate logistic regression results. Firstly, our analysis revealed that female healthcare staff were 2.23 times more likely to have distress symptoms $(\mathrm{OR}=2.23$; 95\% CI: 1.02 to 4.86; $\mathrm{p}=0.043)$ than their male counterparts. Highly educated healthcare staff experienced a higher level of distress $(\mathrm{OR}=1.63 ; 95 \%$ CI: 1.05 to $2.44 ; p=0.017)$ than less-educated staff. Compared to healthcare staff who were not infected by COVID-19, those who were unsure if they were infected by the virus hada higher level of distress $(\mathrm{OR}=3.23 ; 95 \%$ CI: 1.59 to $6.60 ; \mathrm{p}=0.001)$ and depression $(\mathrm{OR}=2.42 ; 95 \%$ CI: 1.33 to $4.37 ; \mathrm{p}=0.004)$. The number of COVID-19 positive cases among healthcare workers' colleagues or friends also positively predicted anxiety symptoms $(\mathrm{OR}=2.11 ; 95 \% \mathrm{CI}: 1.11$ to $4.01 ; \mathrm{p}=0.023)$. Compared to radiology technologists- the most common job function category in this study, medical doctors were less likely to experience distress $(\mathrm{OR}=0.09 ; 95 \% \mathrm{CI}: 0.02$ to 0.53 ; $\mathrm{p}=0.007)$ and anxiety symptoms $(\mathrm{OR}=0.12 ; 95 \% \mathrm{CI}: 0.03$ to $0.48 ; \mathrm{p}=0.003)$ while technicians $(\mathrm{OR}=0.27 ; 95 \% \mathrm{CI}$ : 0.08 to $0.89 ; \mathrm{p}=0.031)$ and obstetrics staff $(\mathrm{OR}=0.21,95 \%$ CI: 0.06 to $0.81 ; \mathrm{p}=0.023$ ) were less likely to experience anxiety symptoms. Our analysis also showed that healthcare staff's existing chronic health issues and the type of healthcare organization they worked in (private or public) did not have a significant relationship with distress, depression, and anxiety symptoms. The significant results are illustrated in Figure 1.

Secondly, the results demonstrated significant interaction terms between the healthcare workers' age and their number of working days on distress $(\mathrm{OR}=1.03 ; 95 \% \mathrm{CI}$ : 1.00 to $1.06 ; p=0.047)$ and depression symptoms $(\mathrm{OR}=1.03,95 \% \mathrm{CI}: 1.00$ to $1.06 ; p=0.026)$. Figure 1 


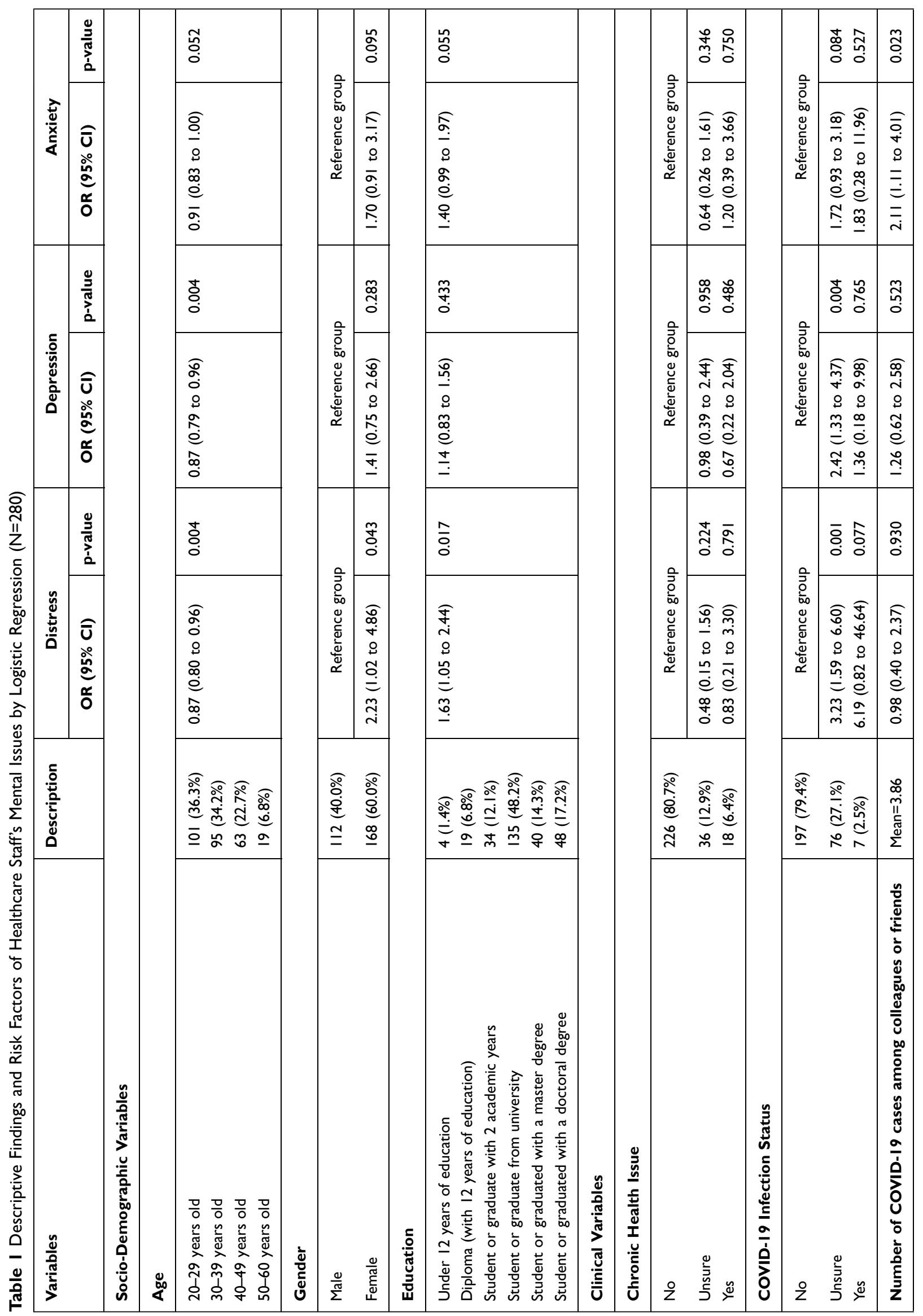




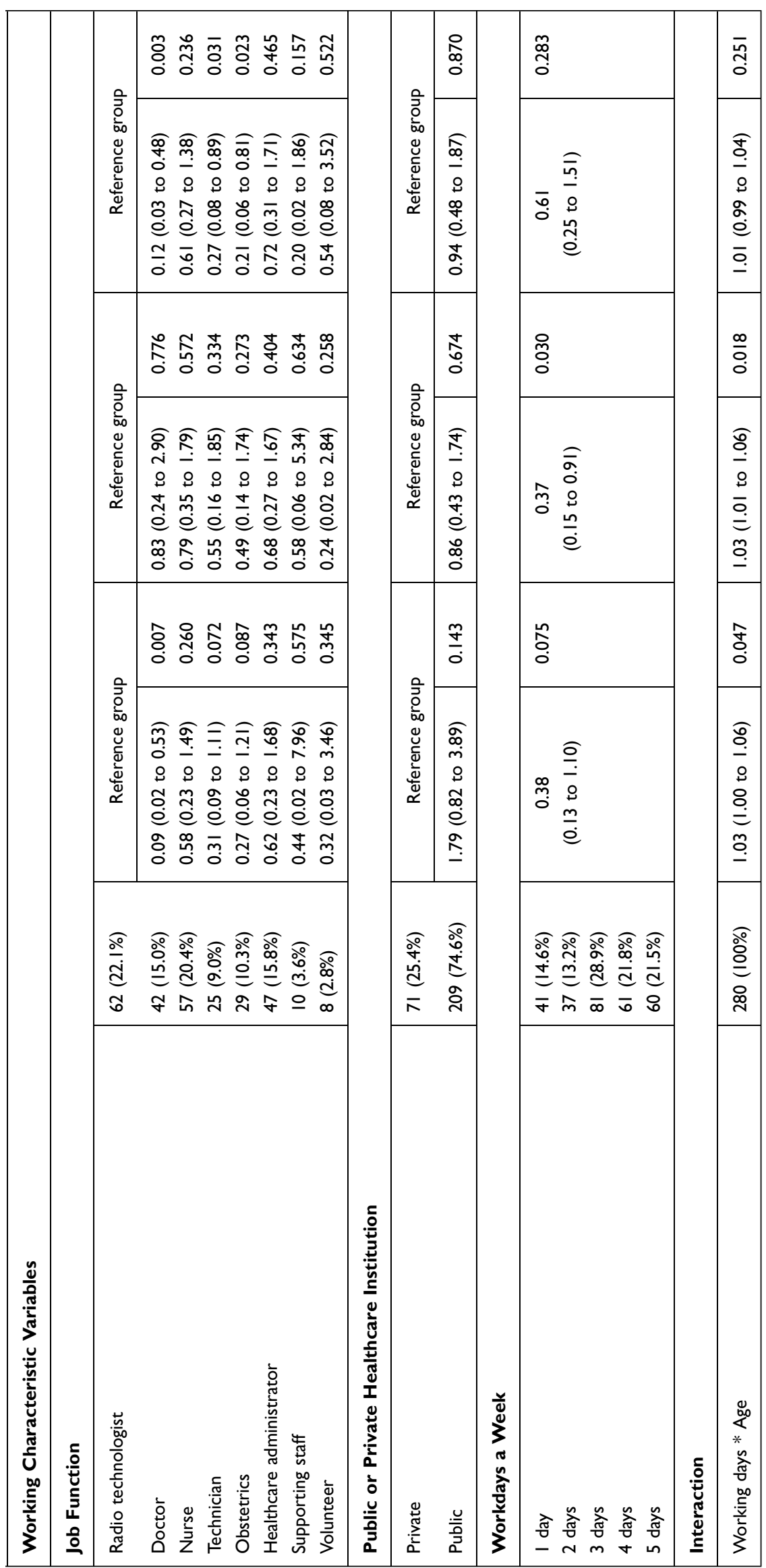




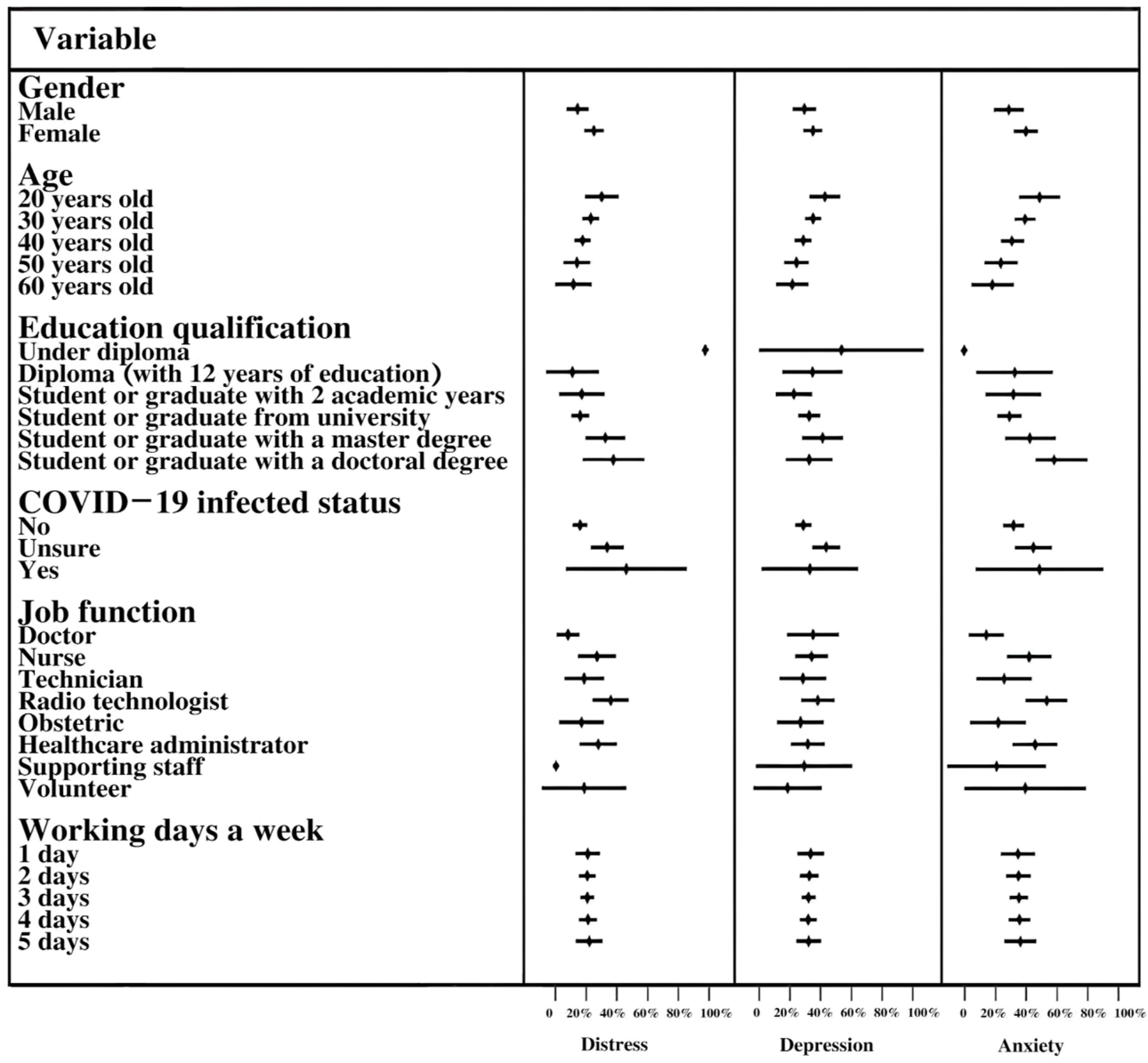

Figure I The predicted odds ratios and their $95 \%$ confidence intervals (Cls) of distress, depression, and anxiety by healthcare staff's socio-demographic, clinical and workrelated characteristics.

illustrates the interaction effect. Further margin analysis on the interaction results revealed that the healthcare staff who were 20 years old and worked only one day per week had the highest likelihood of distress (41\%; 95\% CI: $23 \%$ to $65 \% ; p=0.000)$ and depression symptoms (61\%; $95 \%$ CI: $40 \%$ to $82 \% ; p=0.000)$. In contrast, the healthcare staff who were 20 years old but worked five days a week had a much lower likelihood of having distress $(19 \%$; $95 \% \mathrm{CI}: 19 \%$ to $36 \% ; p=0.023)$ and depression symptoms (24\%; 95\% CI: $7 \%$ to $40 \%$; $p=0.004)$. The chance of having distress and depression symptoms varied significantly by the number of working days among younger participants but not their older counterparts. These margin analysis results are presented in Figure 2.

\section{Discussion}

The findings of our study revealed that nearly a third of the healthcare staff in Iran reported symptoms of distress, depression, and anxiety during the COVID-19 pandemic. Gender and education were significant risk factors to predict the distress of healthcare staff. The participants who were unsure if they were infected with the COVID19 virus reported more distress and depression. Our survey also suggested that the number of COVID-19 cases among a healthcare worker's colleagues or friends positively predicted the worker's anxiety. We also uncovered that healthcare staff's age and their number of working days per week are significantly related to distress and depression symptoms.

Some demographic risk factors found in previous research failed to predict the mental health of the healthcare staff in our study in a significant manner. While gender predicted the mental health among healthcare staff during the COVID-19 pandemic in China, ${ }^{25-27}$ Italy, ${ }^{28}$ Brazil $^{21}$ and several other countries, ${ }^{29}$ it was not significant in this study. Previous studies indicated those who had chronic health issues had worse mental health, ${ }^{30,31}$ but such a relationship was not significant in this study. These findings may indicate that the predictors of the mental health of healthcare staff during the pandemic may vary from one country to 


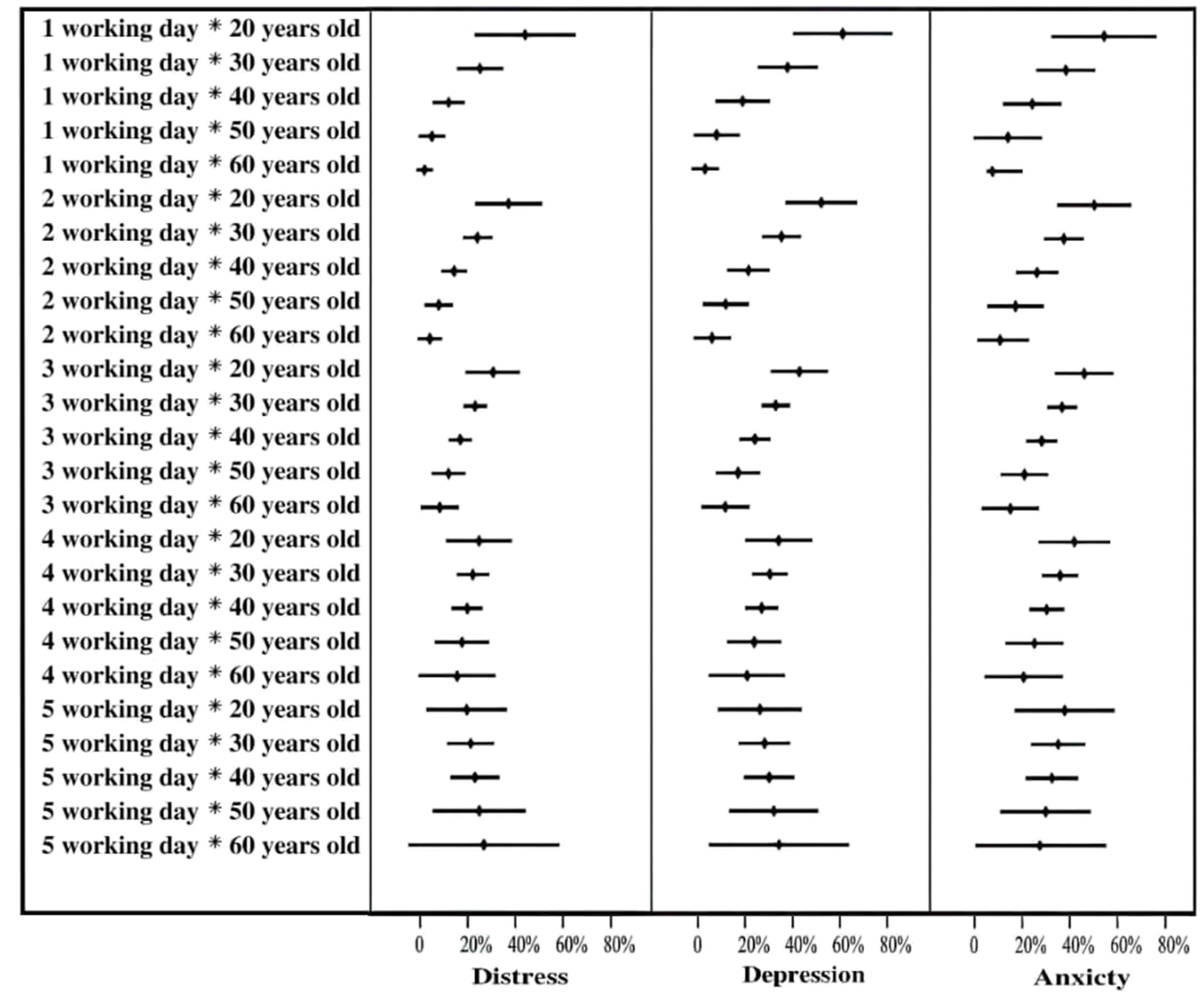

Figure 2 The predicted odds ratios and their $95 \%$ confidence intervals (Cls) of distress, depression, and anxiety by healthcare staff's age and number of workdays a week.

another. ${ }^{32-34}$ Our study also revealed that doctors were more likely to suffer from distress and anxiety than radiology technologists. Prior mental health research among healthcare workers has rarely examined the differences in their mental health by their job functions. Our findings that that radiologists were more likely to experience anxiety in their workplace might be specific to the Iranian context, as there is heavy shortage of radiologists in Iran. ${ }^{35}$ We suggest future research on mental health under COVID-19 pandemic to investigate furtherthe difference in mental health by healthcare workers' job functions in different contexts.

Moreover, this study, to our best knowledge, is the first to uncover the interaction effect between age and working days in the identification of mentally vulnerable healthcare workers. Previous literature has uncovered age as a predictor in a linear relationship only. ${ }^{2}$ Our findingof an interaction effect suggests that the use of individual healthcare workers' age as a predictor of mental health depends on their number of working days a week. The linear relationship that older healthcare workers are less likely to have mental health issues (the previous finding) is significant only among those who had fewer working days per week. Hence, our findings suggest important boundary conditions on the use of age as a predictor of mental health. Moreover, the obtained significant interaction effect indicates it is important to consider age, working days, and their interaction effect together in future predictive models of mental health among healthcare staff. Specifically, our findings revealed that younger healthcare staff who did not work many days and older healthcare staff who worked more days are the most mentally vulnerable groups. Unlike previous studies that have shown that younger individuals experienced greater mental issues in SARS, ${ }^{36}$ we showed younger healthcare staff did not generally have worse mental health, and therefore better predictive models need to take account of their working days to jointly predict the mental health of healthcare staff during the pandemic to better identify which healthcare workers might need more help.

\section{Limitations}

Our study has several limitations that open new agendas for future investigations. While we sampled from all the provinces of Iran, our sample did not aim to capture healthcare staff by their proportions based on the provinces in Iran. Future studies may use more representative samples from more countries in the world. ${ }^{1}$ We conducted 
this study using an observational cross-sectional survey, so our findings are predictive instead of causal. Lastly, even though our sample contained adults who reported having COVID-19, the number of infected cases in our sample is too small, and we suspect they would belong to the majority of COVID-19 infected cases that have mild symptoms, as people with more severe symptoms would not have been able to answer our survey.

\section{Conclusions}

Our identification of the demographic, clinical, and workrelated characteristics as risk factors of mental health issues for healthcare staff during the COVID-19 crisis enables psychological services to better identify mentally venerable healthcare staff. The interaction of healthcare staff's age and the number of working days a week suggests important boundary conditions and room for improvement in the identification models applied in the prior studies. Our results suggest the need to further investigate the work-related characteristics during the COVID19 pandemic as a fruitful direction to identify mentally vulnerable healthcare staff during the COVID-19 crisis.

\section{Acknowledgments}

This work was supported by Tsinghua University-INDITEX Sustainable Development Fund (No. TISD201904).

\section{Author Contributions}

All authors contributed to data analysis, drafting or revising the article, have agreed on the journal to which the article will be submitted, gave final approval of the version to be published, and agree to be accountable for all aspects of the work.

\section{Disclosure}

The authors report no conflicts of interest for this work.

\section{References}

1. Rajkumar RP. COVID-19 and mental health: a review of the existing literature. Asian $J$ Psychiatr. 2020;52:102066. doi:10.1016/j. ajp.2020.102066

2. Braquehais MD, Vargas-Cáceres S, Gómez-Durán E, et al. The impact of the COVID-19 pandemic on the mental health of healthcare professionals. QJM. 2020:1-5. doi:10.1093/qjmed/hcaa207.

3. Muller AE, Hafstad EV, Himmels JPW. The mental health impact of the COVID-19 pandemic on healthcare workers and interventions to help them: a rapid systematic review. Psychiatry Res. 2020;293:113441. doi:10.1016/j.psychres.2020.113441

4. Shaukat N, Ali DM, Razzak J. Physical and mental health impacts of COVID-19 on healthcare workers: a scoping review. Int J Emerg Med. 2020;13:40. doi:10.1186/s12245-020-00299-5
5. Vizheh M, Qorbani M, Arzaghi SM, Muhidin S, Javanmard Z, Esmaeili M. The mental health of healthcare workers in the COVID-19 pandemic: a systematic review. $J$ Diabetes Metab Disord. 2020;19(2):1967-1978. doi:10.1007/s40200-020-00643-9

6. Spoorthy MS, Pratapa SK, Mahant S. Mental health problems faced by healthcare workers due to the COVID-19 pandemic: a review. Asian J Psychiatr. 2020;51:102119. doi:10.1016/j.ajp.2020.102119

7. Woolley K, Smith R, Arumugam S. Personal Protective Equipment (PPE) guidelines, adaptations and lessons during the COVID-19 pandemic. Ethics Med Public Health. 2020;14:100546. doi:10.1016/j.jemep.2020.100546

8. Lee S. Coronavirus Anxiety Scale: a brief mental health screener for COVID-19 related anxiety. Death Stud. 2020;44:1-9. doi:10.1080/ 07481187.2020.1748481

9. Roy D, Tripathy S, Kar SK, Sharma N, Verma SK, Kaushal V. Study of knowledge, attitude, anxiety \& perceived mental healthcare need in Indian population during COVID-19 pandemic. Asian J Psychiatr. 2020;51:102083. doi:10.1016/j.ajp.2020.102083

10. Jahanshahi AA, Dinani MM, Madavani AN, et al. The distress of Iranian adults during the Covid-19 pandemic - more distressed than the Chinese and with different predictors. Brain Behav Immun. 2020;87:124-125. doi:10.1016/j.bbi.2020.04.081

11. Ruiz M Italian nurse who fell ill commits suicide over fears of spreading coronavirus, the nursing group says. 2020. Available from: https://www.foxnews.com/world/italian-nurse-suicide-fearsspreading-coronavirus. Accessed April 8, 2021.

12. Lai J, Ma S, Wang Y, et al. Factors associated with mental health outcomes among health care workers exposed to coronavirus disease 2019. JAMA Net Open. 2020;3:e203976. doi:10.1001/jamanet workopen.2020.3976

13. Takian A, Raoofi A, Kazempour-Ardebili S. COVID-19 battle during the toughest sanctions against Iran. Lancet. 2020;2019:30668.

14. Zandifar A, Badrfam R. Iranian mental health during the COVID-19 epidemic. Asian J Psychiatr. 2020;51:101990. doi:10.1016/j. ajp. 2020.101990

15. Byrne L. A role for lived experience mental health leadership in the age of Covid-19. J Mental Health. 2020;29:1-4. doi:10.1080/ 09638237.2020 .1766002

16. Henry BM, Lippi G. Chronic kidney disease is associated with severe coronavirus disease 2019 (COVID-19) infection. Int Urol Nephrol. 2020;52(6):1193-1194. doi:10.1007/s11255-020-02451-9

17. Banerjee D. Age and ageism in COVID-19': elderly mental healthcare vulnerabilities and needs. Asian J Psychiatr. 2020;51:102154. doi:10.1016/j.ajp.2020.102154

18. Chen J, Zhang SX, Wang Y, et al. The curvilinear relationship between the age of adults and their mental health in Iran after its peak of COVID-19 cases. MedRxiv. 2020;2020(6):20128132. doi:10.1101/2020.06.11.20128132

19. Chen X, Zhang S, Afshar Jahanshahi AA, et al. Belief in conspiracy theory about COVID-19 predicts mental health and well-being - a study of healthcare staff in Ecuador. medRxiv. 2020. doi:10.1101/ 2020.05.26.20113258

20. Zhang SX, Graf-Vlachy L, Su R, Li J, Looi KH. Who do not wash their hands during the Covid-19 pandemic? Social media use as a potential predictor. MedRxiv. 2020;06:20119230. doi:10.1101/ 2020.06.01.20119230

21. Zhang SX, Wang Y, Jahanshahi AA, Li J, Schmitt VGH. Early evidence and predictors of mental distress of adults one month in the COVID-19 epidemic in Brazil. J Psychosom Res. 2021;142. doi:10.1101/2020.04.18.20070896.

22. Try as it may, Iran can't ban social media platforms. Available from: https://www.bangkokpost.com/opinion/opinion/1609122/try-as-it-mayiran-cant-ban-social-media-platforms. Accessed January 11, 2019.

23. Fawad M, Rawashdeh F, Parmar P, Ratnayake R. Simple ideas to mitigate the impacts of the COVID-19 epidemic on refugees with chronic diseases. Confl Health. 2020;14. doi:10.1186/s13031-02000277-x. 
24. Ahmadi SM, Masjedi AA, Bakhtiari M, Davazdah Emamy MH. Psychometric properties of Persian version of patient health questionnaires-4 (PHQ-4) in coronary heart disease patients. Iran $J$ Psychiatric Behav Sci. 13(4). doi:10.5812/ijpbs. 85820

25. Li H, Wang S, Zhong F, et al. Age-dependent risks of incidence and mortality of COVID-19 in Hubei Province and other parts of China. Front Med. 2020;7:190. doi:10.3389/fmed.2020.00190

26. Xiao X, Zhu X, Fu S, Hu Y, Li X, Xiao J. Psychological impact of healthcare workers in China during COVID-19 pneumonia epidemic: a multi-center cross-sectional survey investigation. $J$ Affect Disord. 2020;274:405-410. doi:10.1016/j.jad.2020.05.081

27. Wang Y, Zhu LY, Ma YF, et al. Association of insomnia disorder with sociodemographic factors and poor mental health in COVID-19 inpatients in China. Sleep Med. 2020;75:282-286. doi:10.1016/j. sleep.2020.06.011

28. Casagrande M, Favieri F, Tambelli R, Forte G. The enemy who sealed the world: effects quarantine due to the COVID-19 on sleep quality, anxiety, and psychological distress in the Italian population. Sleep Med. 2020;75:12-20. doi:10.1016/j.sleep.2020.05.011

29. Zhang SX, Sun S, Jahanshahi AA, et al. Developing and testing a measure of COVID-19 organizational support of healthcare workersresults from Peru, Ecuador, and Bolivia. Psychiatry Res. 2020;291:113174. doi:10.1016/j.psychres.2020.113174

30. Sodhi R, Manju D. Spiritually and mental health among normal and chronic disease group. Int J Res Stud Psychol. 2013;2. doi:10.5861/ ijrsp.2012.106.
31. Hüfner K, Fuchs D, Blauth M, Sperner-Unterweger B. How acute and chronic physical disease may influence mental health - an analysis of neurotransmitter precursor amino acid levels. Psychoneuroen docrinology. 2019;106:95-101. doi:10.1016/j.psyneuen.2019.03.028

32. Zhang SX, Liu J, Afshar Jahanshahi A, et al. At the height of the storm: healthcare staff's health conditions and job satisfaction and their associated predictors during the epidemic peak of COVID-19. Brain Behav Immun. 2020;87:144-146. doi:10.1016/j. bbi.2020.05.010

33. Zhang SX, Sun S, Jahanshahi AA, Wang Y, Madavani AN, Dinani MM. Beyond predicting the number of infections: predicting who is likely to be COVID negative or positive. MedRxiv. 2020;4 . doi:10.1101/2020.04.30.20086348.

34. Tang PM, Zhang SX, Li CH, Wei F. Geographical identification of the vulnerable groups during COVID-19 crisis: the typhoon eye effect and its boundary conditions. MedRxiv. 2020;4:20083667. doi:10.1101/2020.04.28.20083667

35. Yeung D, Fung H. Age differences in coping and emotional responses toward SARS: a longitudinal study of Hong Kong Chinese. Aging Ment Health. 2007;11(5):579-587. doi:10.1080/13607860601086355

36. Esmaeilzadeh H, Doshmangir L, Tafazzoli M. Key factors affecting use of telemedicine technology in Iran: the viewpoints of experts. Cultivate J. 2013;22.03:51-58.
Journal of Multidisciplinary Healthcare

\section{Publish your work in this journal}

The Journal of Multidisciplinary Healthcare is an international, peerreviewed open-access journal that aims to represent and publish research in healthcare areas delivered by practitioners of different disciplines. This includes studies and reviews conducted by multidisciplinary teams as well as research which evaluates the results or conduct of such teams or healthcare processes in general. The journal

\section{Dovepress}

covers a very wide range of areas and welcomes submissions from practitioners at all levels, from all over the world. The manuscript management system is completely online and includes a very quick and fair peer-review system. Visit http://www.dovepress.com/testimonials. php to read real quotes from published authors. 